DOI: $10.15593 /$ RZhBiomeh/2016.3.06

УДК 531/534:[57+61]

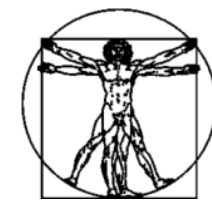

Росеийекий

\title{
ЧИСЛЕННОЕ ИССЛЕДОВАНИЕ НАПРЯЖЕННО-ДЕФОРМИРОВАННОГО СОСТОЯНИЯ ТАЗОБЕДРЕННОГО СУСТАВА ПРИ РОТАЦИОННОЙ ОСТЕОТОМИИ ПРОКСИМАЛЬНОГО УЧАСТКА БЕДРЕННОЙ КОСТИ
}

\author{
О.А. Саченков ${ }^{1}$, Р.Ф. Хасанов ${ }^{2}$, П.С. Андреев ${ }^{2}$, Ю.Г. Коноплев ${ }^{1}$ \\ ${ }^{1}$ Институт математики и механики имени Н.И. Лобачевского Казанского (Приволжского) федерального \\ университета, Россия, 420008, Казань, ул. Кремлевская, 18, e-mail: 4works@bk.ru \\ 2 Республиканская клиническая больница Министерства здравоохранения Республики Татарстан, Россия, \\ 420064, Казань, Оренбургский тракт, 138, e-mail: zaimiche@mail.ru
}

\begin{abstract}
Аннотация. Актуальность данного исследования продиктована высокой частотой встречаемости болезни Легга-Кальве-Пертеса. Она занимает 25-30 \% среди всех заболеваний тазобедренного сустава в детском возрасте. Усложняет ситуацию отсутствие обоснованных рекомендаций к тактике оперативных вмешательств в целом и ротационно-флексионных остеотомий в частности. Целью исследования является определение напряженно-деформированного состояния сустава при различных направлениях и величинах угла ротации. Расчеты проводились с помощью компьютерного моделирования тазобедренного сустава с учетом локализации дегенеративно-дистрофического процесса и тяжести поражения эпифиза у детей и подростков на примере болезни Легга-Кальве-Пертеса. Численные исследования выполнены с помощью метода конечных элементов в пакете Siemens NX. В работе было учтено влияние следующих мышц: $\mathrm{mm}$. piriformis, rectus femoris, iliopsoas, obturatorius internus, gluteus minimus, medius et maximus. Расчеты позволяют определить усилия, возникающие в мышцах, реактивную силу и реактивный момент, действующие в суставе при различных углах ротации (эти величины актуальны для оценки жесткости аппарата внешней фиксации). Были определены максимальные касательные напряжения и напряжения по Мизесу, возникающие в вертлужной впадине и на проксимальном участке бедренной кости. Оценка прочности производилась по максимальным касательным напряжениям. Так, в вертлужной впадине при ротации более $25^{\circ}$ вперед и при ротации более $35^{\circ}$ назад наибольшие величины максимальных касательных напряжений превышают $1,7 \mathrm{MПа;} \mathrm{при} \mathrm{ротации} \mathrm{более} 30^{\circ}$ назад величина аналогичных напряжений превышает $6,4 \mathrm{MПа;} \mathrm{при} \mathrm{ротации} \mathrm{вперед} \mathrm{до} 50^{\circ}$ наибольшие максимальные касательные напряжения достигают величины в 5,0 МПа. Таким образом, при рассмотрении тактики проведения ротации стоит отметить, что при вращении назад на величину угла более $30^{\circ}$ максимальные касательные напряжения в суставе попадают в нижнюю область критических значений, при $35^{\circ}$ - в верхнюю область критических значений. При рассмотрении ротации вперед на угол более $25^{\circ}$ максимальные касательные напряжения попадают в нижнюю область критических значений, перехода через верхнюю область критических значений при ротации до $50^{\circ}$ не происходит.
\end{abstract}

Ключевые слова: болезнь Легга-Кальве-Пертеса, ротационно-фрлексионная остеотомия, математическое моделирование.

(C) Саченков О.А., Хасанов Р.Ф., Андреев П.С., Коноплев Ю.Г., 2016

Саченков Оскар Александрович, к.ф.-м.н., научный сотрудник, Казань

Хасанов Руслан Фаритович, врач травматолог-ортопед, Казань

Андреев Петр Степанович, к.м.н., врач травматолог-ортопед, заведующий травматолого-ортопедическим отделением, Казань

Коноплев Юрий Геннадьевич, д.ф.-м.н., профессор, академик Академии наук Республики Татарстан, заведующий кафедрой теоретической механики, Казань 


\section{ВведЕНИЕ}

Болезнь Легга-Кальве-Пертеса (остеохондропатия головки бедренной кости, в дальнейшем болезнь Пертеса) представляет собой субхондральный некроз ядра окостенения эпифиза головки бедренной кости, сопровождающийся нарушением устойчивости эпифиза при статической и динамической нагрузках [18, 21-24, 29]. Этиология болезни Пертеса до настоящего времени остается дискуссионной, но продолжают обсуждаться травматическая, инфекционная, обменно-гормональная, наследственная, циркуляторная или сосудистая теории, ни одна из которых не располагает достаточно весомыми доказательствами. Тяжесть процесса и его продолжительность определяются, в известной мере, степенью изменений в сосудах капсулы сустава и нижней конечности, а также степенью деструкции эпифиза головки бедренной кости. Трудность диагностики на ранних этапах заболевания и, как следствие этого, неудовлетворенность результатами консервативного лечения детей с болезнью Пертеса заставили ортопедов заняться поисками эффективных способов хирургического лечения. В настоящий момент предложены различные виды оперативных вмешательств при болезни Пертеса: стимулирующие, декомпрессивные и реконструктивные операции на костях таза и бедра.

Хирургический метод лечения остеохондропатии головки бедра у детей (при наличии большого очага деструкции) имеет решающее значение. В основе реконструктивных методов оперативного лечения лежит выведение очага деструкции эпифиза головки бедра из-под нагрузки с помощью деторсионноваризирующих и ротационно-флексионных остеотомий. Пример тактик ротационных остеотомий приведен на рис. 1. В предлагаемой методике Y. Sugioka происходит отсечение проксимального участка и большого вертела (линии рассечения указаны на рис. $1, a$ ), после ротации крепление обеспечивается винтами, при этом подразумевается артротомия. В предлагаемой методике Соколовского происходит отсечение проксимального участка и большого вертела (линии рассечения указаны на рис. 1,6 ), после ротации крепление обеспечивается винтами и скобой, при этом подразумевается артротомия. В предлагаемой методике рассечение проксимального участка производится по линии дуги Адамса (линия рассечения указана на рис. 1, в), фиксация после ротации осуществляется за счет внешнего стержневого аппарата, при этом не подразумевается артротомия, а значит снижается степень инвазии. Разработанные методы лечения предусматривают поворот головки бедра кпереди или кзади на $90^{\circ}$, что зачастую приводит к скручиванию капсулы сустава. Это, в свою очередь, ведет к окклюзии сосудов, питающих тазобедренный сустав и, как следствие этого, ишемии головки бедренной кости.

Целью нашего исследования является определение напряженнодеформированного состояния в суставе при различных направлениях и величинах угла ротации. Расчеты проводились с помощью компьютерного моделирования тазобедренного сустава на основе пространственной патологической ориентации проксимального отдела бедренной кости с учетом степени и тяжести поражения, локализации дегенеративно-дистрофического процесса у детей и подростков с болезнью Легга-Кальве-Пертеса. 


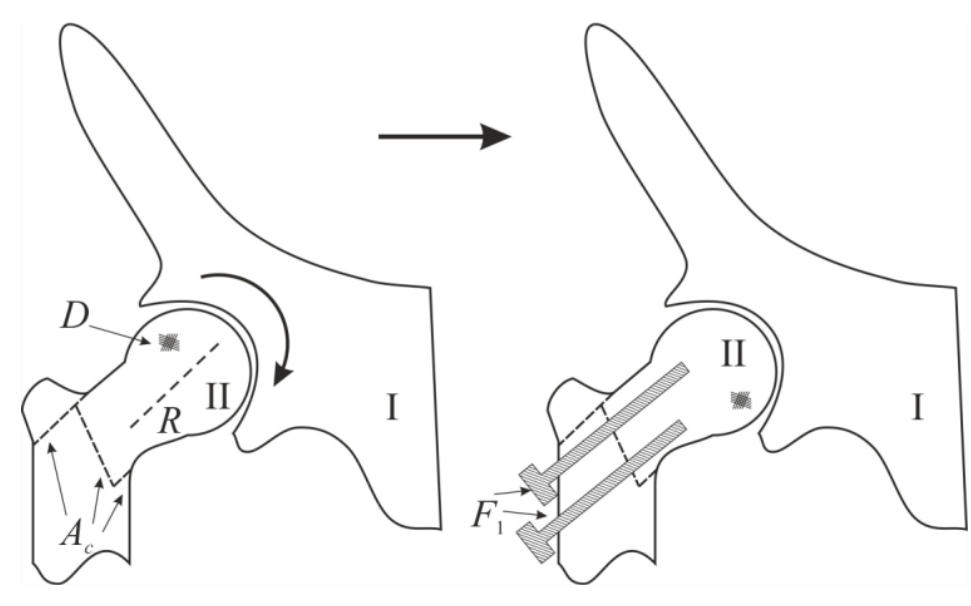

$a$

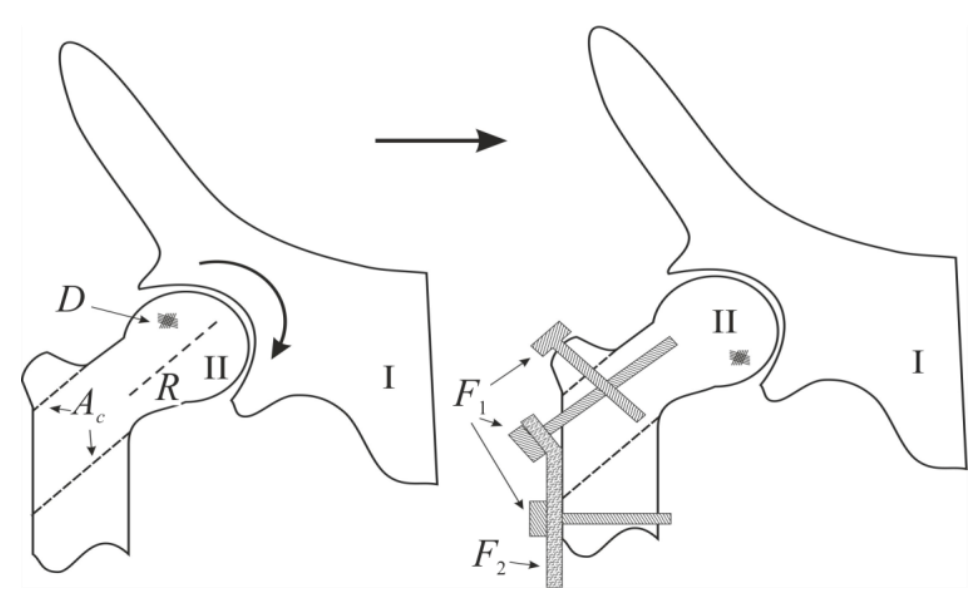

$\sigma$

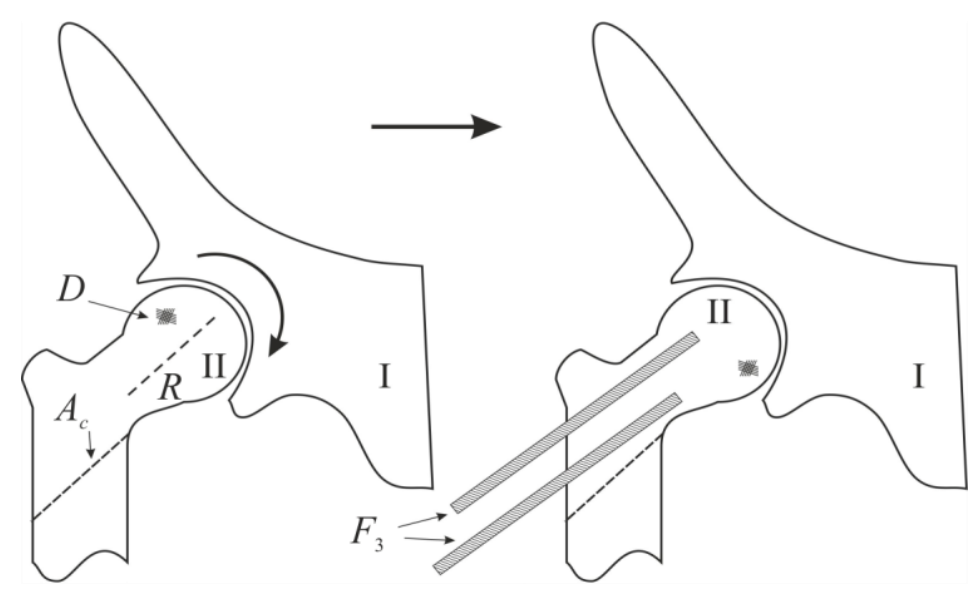

B

Рис. 1. Ротационно-флексионная остеотомия проксимального отдела бедренной кости: $a$ - операция Y. Sugioka (1978); $\sigma$ - операция А.М. Соколовского (1982); в - предлагаемый метод. I - кость таза; II - бедренная кость; $D$ - пораженная область; $R$ - ось ротации; $A_{c}$ - поверхность рассечения; $F_{1}-$ винт; $F_{2}$ - скоба; $F_{3}$ - стержень 


\section{МАТЕРИАЛЫ И МЕТОДЫ ИССЛЕДОВАНИЯ}

\section{Постановка задачи}

При проведении ротационной остеотомии бедренная кость рассекается параболической поверхностью по дуге Адамса и с помощью аппарата внешней фиксации производится ротация вертлужного компонента относительно оси $R$ (см. рис. 2), при этом вследствие удлинения и укорочения мышц происходит перераспределение сил в суставе. При исследовании напряженно-деформированного состояния тазобедренного сустава при ротационной остеотомии в первую очередь был определен список основных мышц, которые оказывают главное влияние: mm. piriformis, rectus femoris, iliopsoas, obturatorius internus, gluteus minimus, medius et maximus [5, 14]. Для моделирования была использована построенная авторами параметрическая модель тазобедренного сустава, более подробное ее описание представлено в работах $[5,12]$, параметрами в этом случае задавались анатомические характеристики сустава и бедренной кости: ацебулярный угол, угол Виберга, шеечно-диафизарный угол, длина проксимального участка бедренной кости, диаметр вертлужной впадины и головки бедренной кости.

Для формулировки задачи напряженно-деформированного состояния тазобедренного сустава были введены следующие упрощения: материал таза и бедренной кости считался изотропным и подчиняющимся закону Гука. Ввиду того, что ротация для ткани происходит мгновенно, для мышц также применим закон Гука, но при этом мышцы работают только на растяжение, оценка последующей релаксации может быть оценена отдельно. На поверхности рассечения и по поверхности вертлужной впадины задавалось условие контакта без трения, при этом выбирались поверхности, на которых может быть реализовано контактное взаимодействие, конфигурация зоны контакта определялась при расчетах. Ротация моделировалась кинематическим нагружением. Фиксирующие элементы были исключены из расчетов.

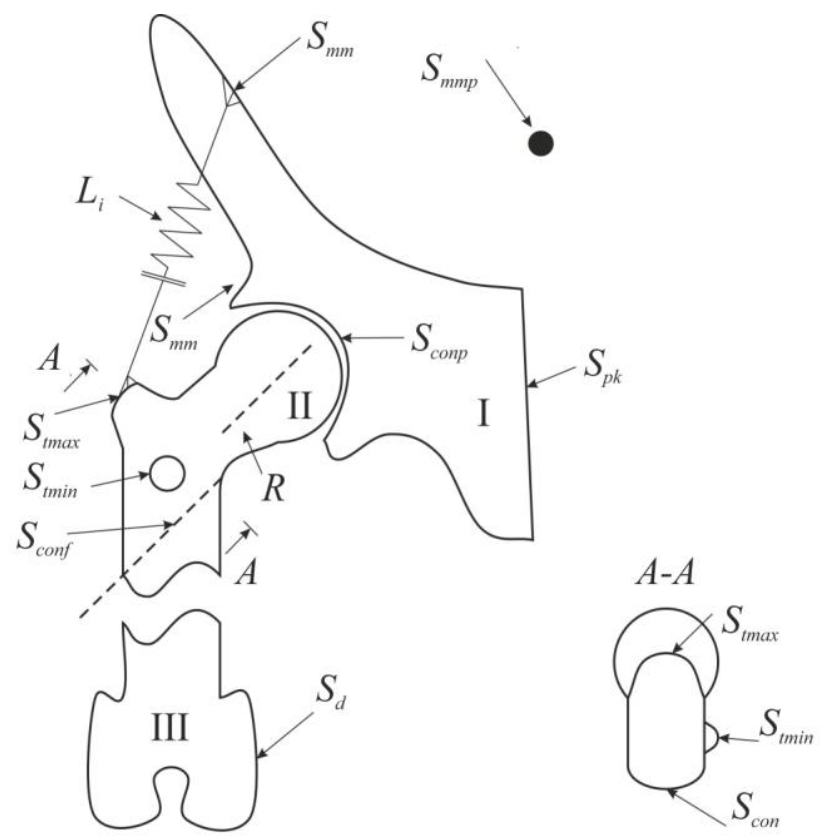

Рис. 2. Расчетная схема: I - объем кости таза; II - объем отсекаемой части бедренной кости; III - объем оставшейся части бедренной кости; $S_{m m p}$ - точки крепления мышц; $S_{m m}$ - область крепления мышц; $S_{t \max }$ - поверхность большого вертела; $S_{t \min }$ - поверхность малого вертела; $S_{d}$ - поверхность дистального участка; $S_{c o n f}-$ поверхность рассечения; $S_{c o n p}-$ суставная поверхность; $S_{p k}-$ поверхность крепления таза к крестцу; $R$ - ось ротации; $L_{i}$ - мышца 


\section{Математическая модель}

Расчетная область включает три тела: таз - I, проксимальный участок бедренной кости - II, остальная часть бедренной кости - III (см. рис. 2). Обозначим $V_{\text {I }}, V_{\text {III }}, V_{\text {III }}$ объемы соответствующих тел, а $\partial V_{\mathrm{I}}, \partial V_{\mathrm{II}}, \partial V_{\mathrm{III}}-$ границы этих множеств (свободную поверхность), $V=V_{\mathrm{I}} \cup V_{\text {II }} \cup V_{\text {III. }}$ Механическое поведение системы, занимающей область $V$ в $R^{3}$ с границей $\partial V$, в рамках линейной теории упругости описывается следующей системой уравнений:

$$
\begin{gathered}
\nabla \cdot \tilde{\sigma}=0, \quad \forall x \in V^{\circ}, \\
\tilde{\varepsilon}=\frac{1}{2}\left(\nabla \vec{u}+(\nabla \vec{u})^{T}\right), \quad \forall x \in V^{\circ}, \\
\tilde{\sigma}=\tilde{\tilde{E}}: \tilde{\varepsilon}, \quad \forall x \in V^{\circ},
\end{gathered}
$$

где $\tilde{\sigma}$ - тензор напряжений; $V=V \cup \partial V ; \tilde{\varepsilon}-$ тензор упругой деформации; $\vec{u}-$ вектор перемещений; $\tilde{\tilde{E}}$ - тензор упругих свойств. Эта постановка выполняется для всех трех тел.

Рассмотрим граничные условия. Для этого введем набор поверхностей. Обозначим за $S_{m m}$ - поверхности на теле I, к которым будут крепиться мышцы, их положение и размер были определены согласно анатомическому атласу, для мышц, которые крепятся к костям, отсутствующим в модели (например к крестцу), определим

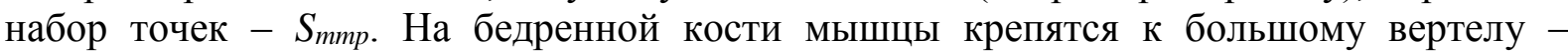
поверхность $S_{\text {tmax }}$ и малому вертелу - поверхность $S_{\text {tmin }}$, а также к дистальному участку поверхность $S_{d}$. Контактное взаимодействие тел возможно на двух поверхностях:

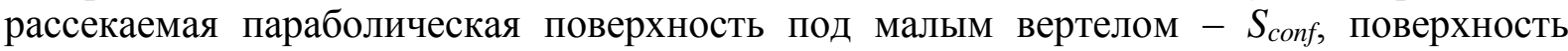

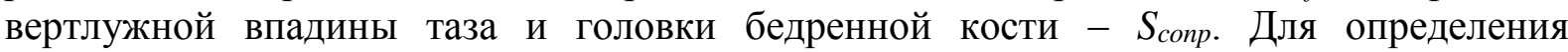
кинематических граничных условий введем поверхность $S_{p k}-$ поверхность таза, которая крепится к крестцу, поверхность вращения $S_{r o t}-$ есть внешняя поверхность проксимального участка бедренной кости за вычетом рассекаемой поверхности, $S_{r o t}=\partial V_{\text {II }} / S_{c o n f} ; \partial V_{\text {III }}$ - внешняя поверхность тела $V_{\text {III. }}$ Граничные условия в этом случае задаются соотношениями

$$
\begin{gathered}
\vec{u}=0, \forall x \in \partial V_{\mathrm{III}} \cup S_{p k}, \\
\vec{u}=f(x, y, z), \quad \forall x \in S_{r o t},
\end{gathered}
$$

где функция $f(x, y, z)$ определяет вращение $S_{r o t}$ относительно оси $R$.

Физические соотношения для мышц использовались в виде

$$
\sigma_{j}=E_{j} \cdot \varepsilon_{j} \frac{1+\operatorname{sign} \varepsilon_{j}}{2}, \quad \forall x \in L_{i},
$$

где $L_{i}$ - прямая, определяющая положение $i$-й мышцы, при этом крепление всех мышц установлено в выделенных ранее областях, все мышцы вводились согласно анатомическому атласу (чтобы не загромождать рис. 2, приведена иллюстрация только для одной мышцы). Здесь $\sigma_{j}$ и $\varepsilon_{j}$ - нормальные напряжения и деформации, возникающие в мышце. Мышцы определялись упругим законом, при работе на растяжение $E_{i}-$ приведенный модуль упругости $i$-й мышцы.

Условие контакта между областями I и II и областями II и III соответственно определяется выражениями 


$$
\begin{aligned}
& \left.\underset{\mathrm{II}}{\left(\vec{r}^{0}-\overrightarrow{\mathrm{r}}^{0}\right) \vec{n}}=\underset{\mathrm{II}}{(\vec{u}-\vec{u}}\right) \vec{n}, \quad \forall x \in S_{\text {conp }}, \\
& \left(\vec{r}_{\mathrm{III}}^{0}-\vec{r}_{\mathrm{II}}^{0}\right) \vec{n}=\left(\vec{u}_{\mathrm{III}}-\vec{u}_{\mathrm{II}}\right) \vec{n}, \quad \forall x \in S_{\text {conf } i} .
\end{aligned}
$$

Для точек, вошедших в контакт, выполняются условия $(7,8)$. В этих выражениях $\vec{r}^{0}$ - начальное положение, а $\vec{u}$ - перемещение точек контактирующих поверхностей, $\vec{n}$ - нормаль к поверхности в точке. На поверхности контакта тела взаимодействуют друг с другом, что приводит к возникновению нормального давления

$$
\vec{t}_{n}=-t_{n} \vec{n}, t_{n}>0, \quad \forall x \in S_{\text {conp }} \cup S_{\text {conf }} .
$$

Используемая методика расчета контактного взаимодействия подробно приведена в работах [12, 19, 26, 27].

\section{Численное решение задачи}

Для проведения численных исследований на основе параметрической модели была построена конечно-элементная модель (рис. 3). Для дискретизации объемов $V_{\mathrm{I}}, V_{\mathrm{II}}$, $V_{\text {III }}$ использовался четырехузловой тетраэдральный конечный элемент с линейной аппроксимацией, характеристики костной ткани брались равными $E=6$ ГПа, $v=0,3$ $[1,12]$.

Для моделирования мышц использовался одномерный конечный. Для определения приведенного модуля Юнга использовалась формула [9]

$$
E \approx 1,5 \frac{F_{\max }}{S},
$$

где $F_{\max }$ - максимальная изометрическая сила мышцы, $S$ - значение анатомического поперечника.

Согласно опубликованным исследованиям по определению максимальной изометрической силы мышцы [4, 15-17] был найден модуль Юнга для мышц, который составил 43-44 кПа.

Для моделирования ротации вводилась дополнительная система координат, ось $z$ которой совпадала с направлением оси вращения (прямая $R$ на рис. $3, \sigma$ ), в этой системе координат задавались кинематические граничные условия поворота для поверхности $\partial V_{\text {II. }}$

Задавался угол мгновенной ротации, после чего осуществлялся расчет модели в несколько этапов. На первом этапе были проведены расчеты в диапазоне угла ротации от $-50^{\circ}$ до $50^{\circ}$, где знак минус означает поворот назад. В результате определялись удлинения и усилия в мышцах, контактное давление в контактирующих зонах $[6,8,28]$. На втором этапе производилось уточнение напряженно-деформированного состояния таза и проксимального участка бедренной кости, для этого рассматривалась отдельно модель таза и проксимального участка бедренной кости, к которой прикладывались силы, возникающие в мышцах ввиду ротации.

При оценке напряженно-деформированного состояния костной ткани было использовано два критерия прочности:

$$
\sigma_{i}<[\sigma], \tau_{\max }<[\tau] .
$$

В расчетах для оценки напряженного состояния костной ткани были приняты

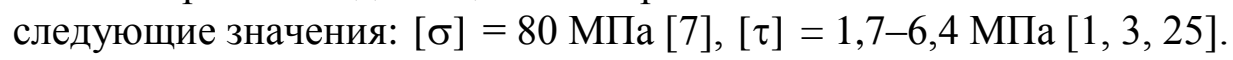




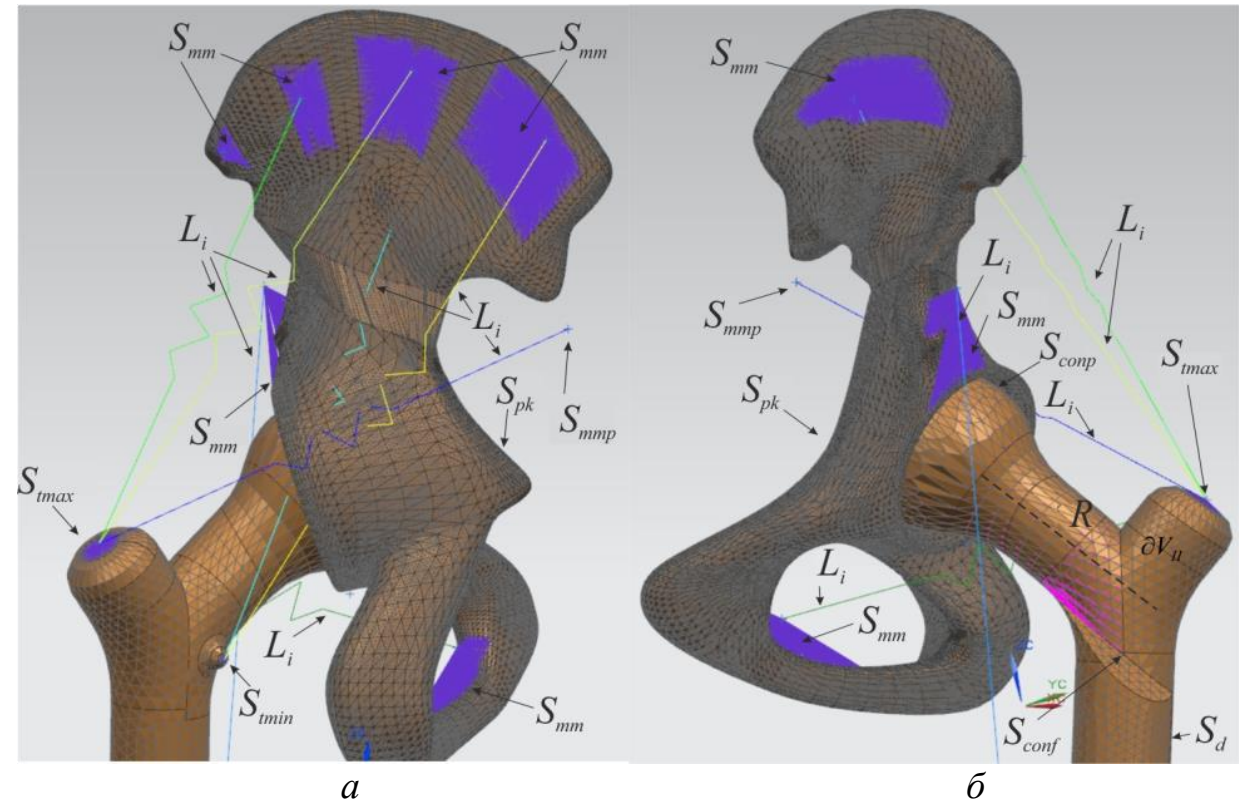

Рис. 3. Конечно-элементная аппроксимация расчетной области: I - объем кости таза; II - объем отсекаемой части бедренной кости; III - объем оставшейся части бедренной кости; $S_{m m p}$ - точки крепления мышц; $S_{m m}$ - область крепления мышц; $S_{t m a x}$ - поверхность большого вертела; $S_{t \min }$ - поверхность малого вертела; $S_{d}$ - поверхность дистального участка; $S_{c o n f}-$ поверхность рассечения; $S_{\text {conp }}$ - суставная поверхность; $S_{p k}$ - поверхность крепления таза к крестцу; $R$ - ось ротации; $L_{i}$ - мышцы

\section{РЕЗУЛЬТАТЫ И ОБСУЖДЕНИЕ}

Были проведены расчеты для величин угла ротации в диапазоне от $-50^{\circ}$ до $50^{\circ}$. Основными величинами для анатомических углов были приняты величины шеечнодиафизарного угла и угла антеторсии, соответствующей здоровой стороне при одностороннем процессе и возрастным нормам при двухстороннем процессе поражения эпифиза. Были определены усилия, возникающие в мышцах, реактивные сила и момент, действующие в суставе при различных углах ротации, которые актуальны для оценки жесткости аппарата внешней фиксации $[10,11,13,20]$. При ротации назад удлинения происходили в mm. iliopsoas, gluteus medius, gluteus maximus, наиболее нагруженной мышцей оказалась $m$. iliopsoas. При ротации вперед удлинения происходили во всех мышцах, наиболее нагруженной мышцей была m. piriformis.

Был проведен анализ напряженно-деформированного состояния вертлужной впадины и проксимального участка бедренной кости при различных углах ротации. Так, при ротации вперед напряжения по Мизесу локализуются в задне-верхнем отделе вертлужной впадины, а также наблюдается небольшая область локализации напряжений в районе центра вертлужной впадины (см. рис. 4, $a, \sigma)$. При ротации назад напряжения по Мизесу также локализуются в задне-верхнем отделе вертлужной впадины, но область локализации больше (см. рис. 4, 6, , ). При ротации до $30^{\circ}$ вперед и назад величины наибольших напряжений по Мизесу в вертлужной впадине отличаются на 10-20\% (4,8 МПа при ротации на $30^{\circ}$ вперед и 3,8 МПа при ротации на $30^{\circ}$ назад). При увеличении ротации вплоть до 50॰ наибольшие напряжения по Мизесу в вертлужной впадине при различных направлениях ротации отличаются на $1 \%$ и возрастают до 6 МПа. 
Так, при вращении вперед максимальные касательные напряжения локализуются в задне-верхнем отделе вертлужной впадины, а также наблюдается небольшая область локализации напряжений в районе центра вертлужной впадины (см. рис. 5, a, б). При вращении назад максимальные касательные напряжения также локализуются в задневерхнем отделе вертлужной впадины, но область локализации больше (см. рис. 5, ,,$~ 2)$. При ротации до $30^{\circ}$ вперед и назад величины наибольших максимальных касательных напряжений в вертлужной впадине отличаются на 10-20 \% (1,8 МПа при ротации на $30^{\circ}$ вперед и 1,5 МПа при ротации на $30^{\circ}$ назад). При увеличении ротации вплоть до $50^{\circ}$ наибольшие максимальные касательные напряжения в вертлужной впадине при различных направлениях ротации отличаются на 1 \% и возрастают до 2,5 МПа.

При анализе величин наибольших максимальных касательных напряжений в вертлужной впадине в зависимости от углов ротации (рис. 6) стоит отметить участки при ротации более $25^{\circ}$ вперед и при ротации более $35^{\circ}$ назад. В этих случаях величины напряжений превышают 1,7 МПа, что согласно исследованиям [1-3] соответствует нижней границе предельных касательных напряжений. На практике индивидуальные характеристики предельных напряжений могут быть уточнены на основе экспериментальных исследований $[1,3]$.

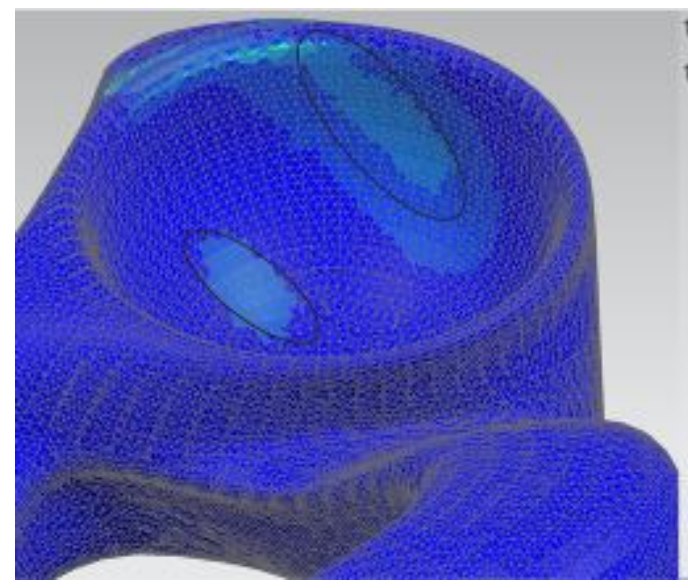

$a$

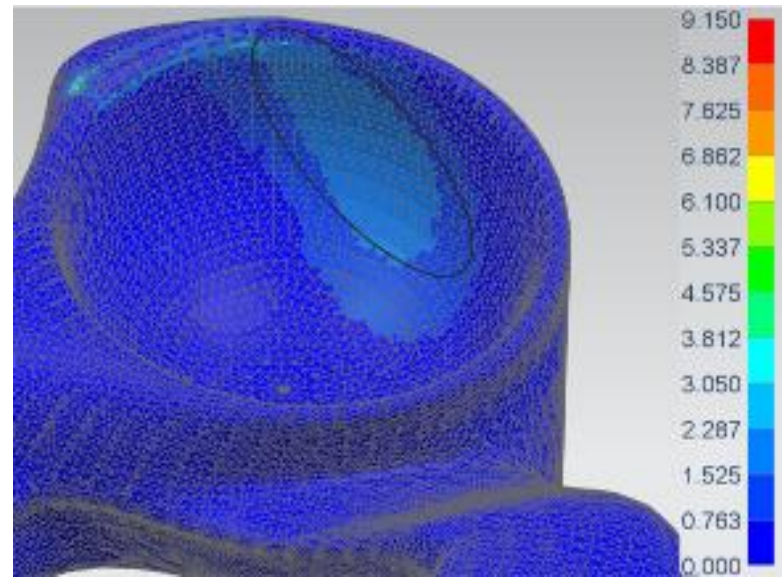

$B$

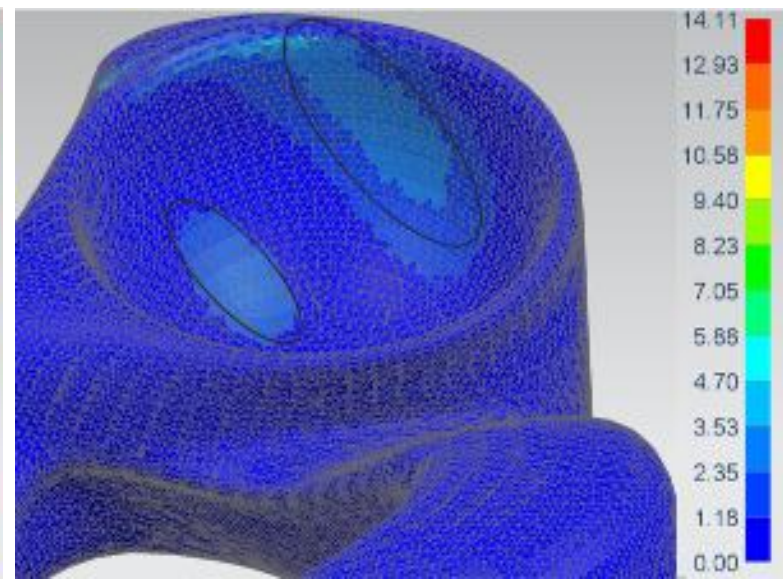

$\sigma$

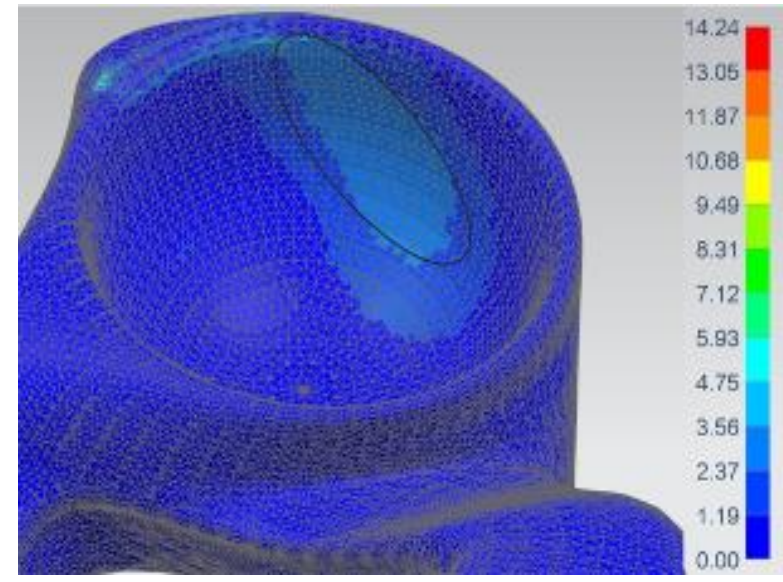

2

Рис. 4. Распределение напряжений по Мизесу, МПа, в вертлужной впадине при различных величинах угла ротации: $a$ - на $30^{\circ}$ вперед; $\sigma$ - на $50^{\circ}$ вперед; 6 - на $30^{\circ}$ назад; 2 - на $50^{\circ}$ назад 


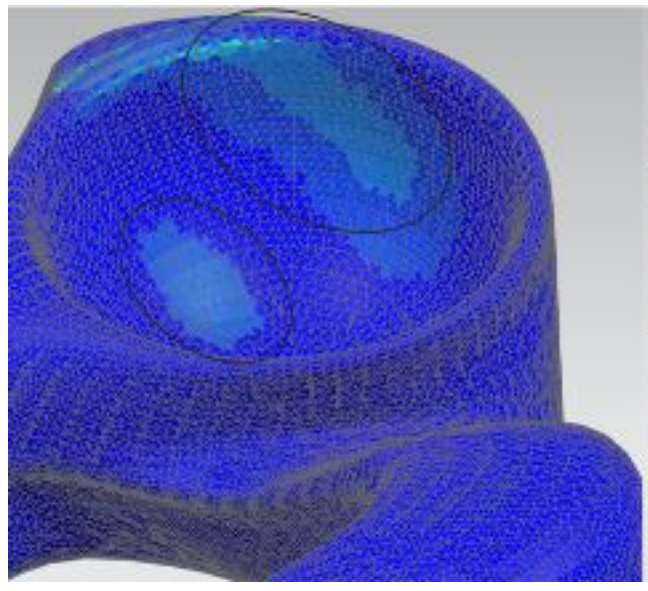

$a$

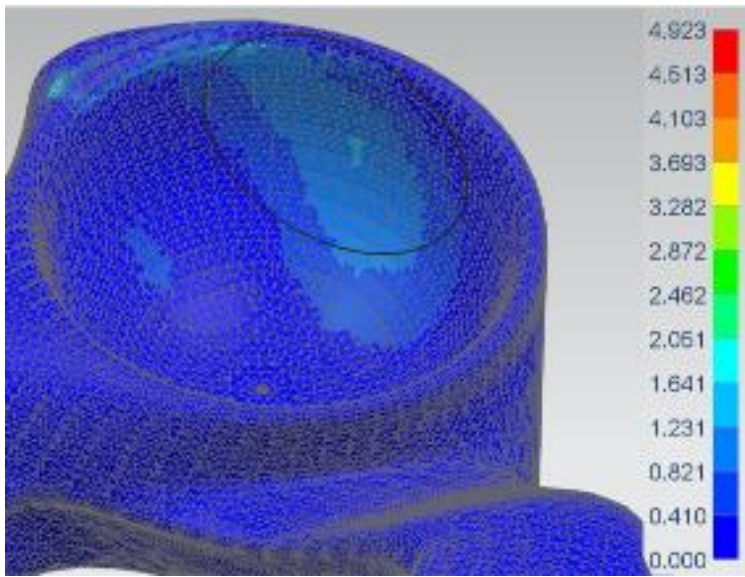

B

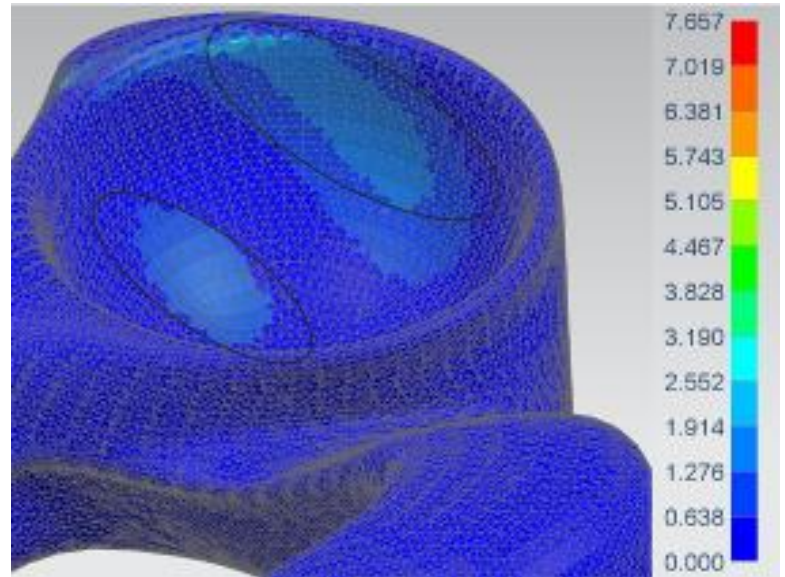

6

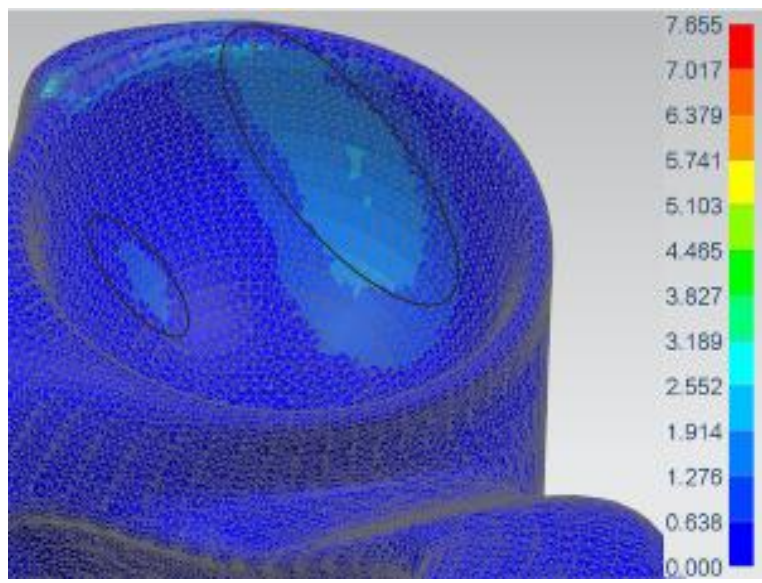

2

Рис. 5. Распределение максимальных касательных напряжений, МПа, в вертлужной впадине при различных величинах угла ротации: $a-$ на $30^{\circ}$ вперед; $\sigma$ - на $50^{\circ}$ вперед; $в$ - на $30^{\circ}$ назад; 2 - на $50^{\circ}$ назад

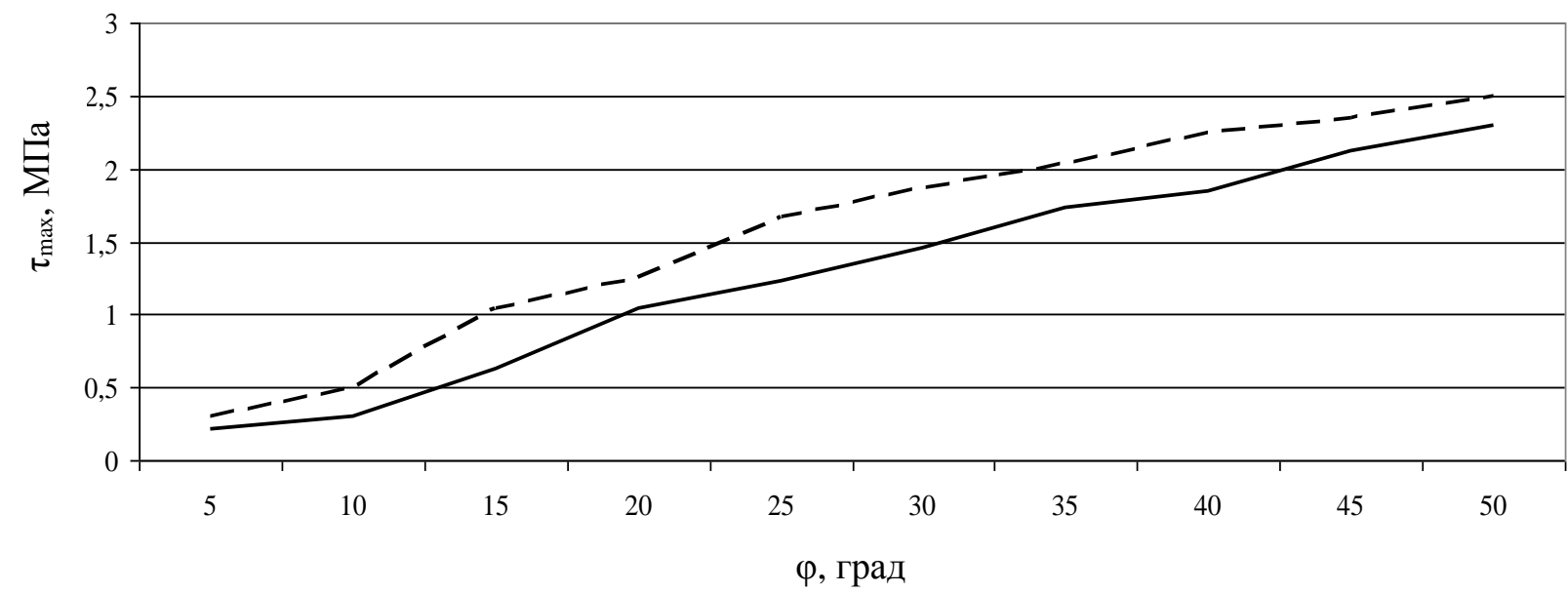

Рис. 6. Величины наибольших максимальных касательных напряжений в вертлужной впадине при различных величинах угла ротации: вращение вперед (пунктирная линия), вращение назад

(сплошная линия) 
Так, при ротации вперед напряжения по Мизесу локализуются в шейке проксимального участка бедренной кости (рис. $7, a, \sigma)$. При ротации назад напряжения по Мизесу также локализуются в шейке проксимального участка бедренной кости, но и образуется концентрация напряжений в области рассечения (рис. $7,6,2$ ). При ротации вплоть до $50^{\circ}$ вперед и назад величины наибольших напряжений по Мизесу в области шейки проксимального участка бедренной кости отличаются на 20-30 \% (6,4 МПа при ротации на $30^{\circ}$ вперед и 8,7 МПа при ротации на $30^{\circ}$ назад).

Так, при ротации вперед максимальные касательные напряжения локализуются в шейке проксимального участка бедренной кости (рис. $8, a$, б). При ротации назад максимальные касательные напряжения также локализуются в шейке проксимального участка бедренной кости, но и образуется концентрация напряжений в области рассечения (см. рис. 8, в, г). При ротации вплоть до $50^{\circ}$ вперед и назад величины наибольших максимальных касательных напряжений в области шейки проксимального участка бедренной кости отличаются на 25-35 \% (3,4 МПа при ротации на $30^{\circ}$ вперед и 5,2 МПа при ротации на $30^{\circ}$ назад).
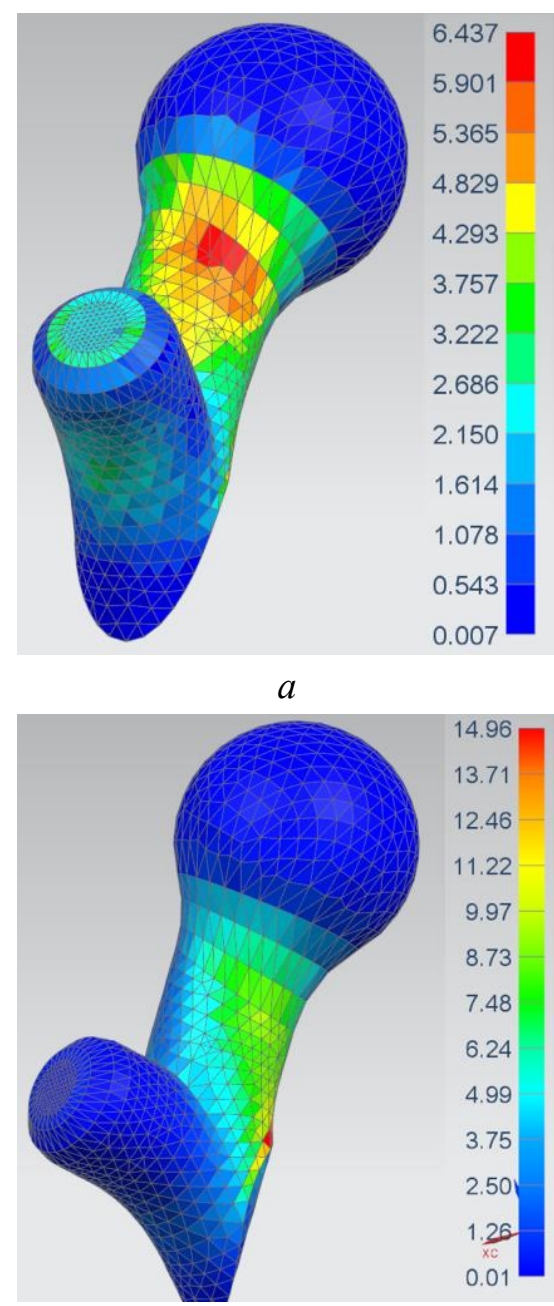

B
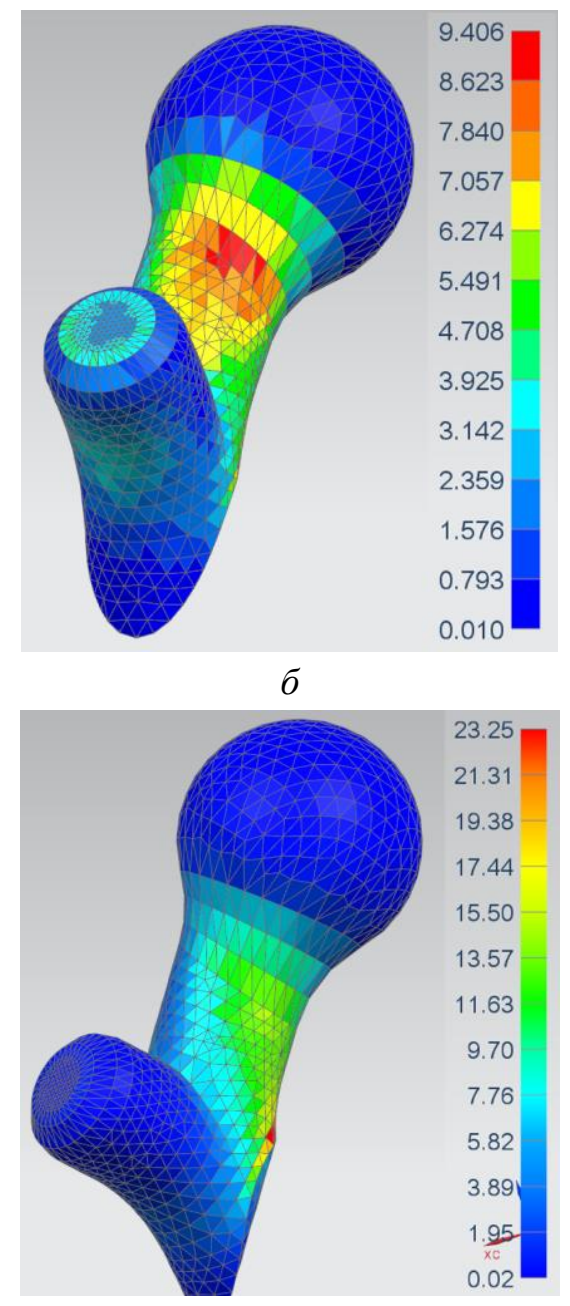

2

Рис. 7. Распределение напряжений по Мизесу; МПа в проксимальном участке бедренной кости при различных величинах угла ротации: $a$ - на $30^{\circ}$ вперед; $\sigma$ - на $50^{\circ}$ вперед; $в$ - на $30^{\circ}$ назад; г- на $50^{\circ}$ назад 

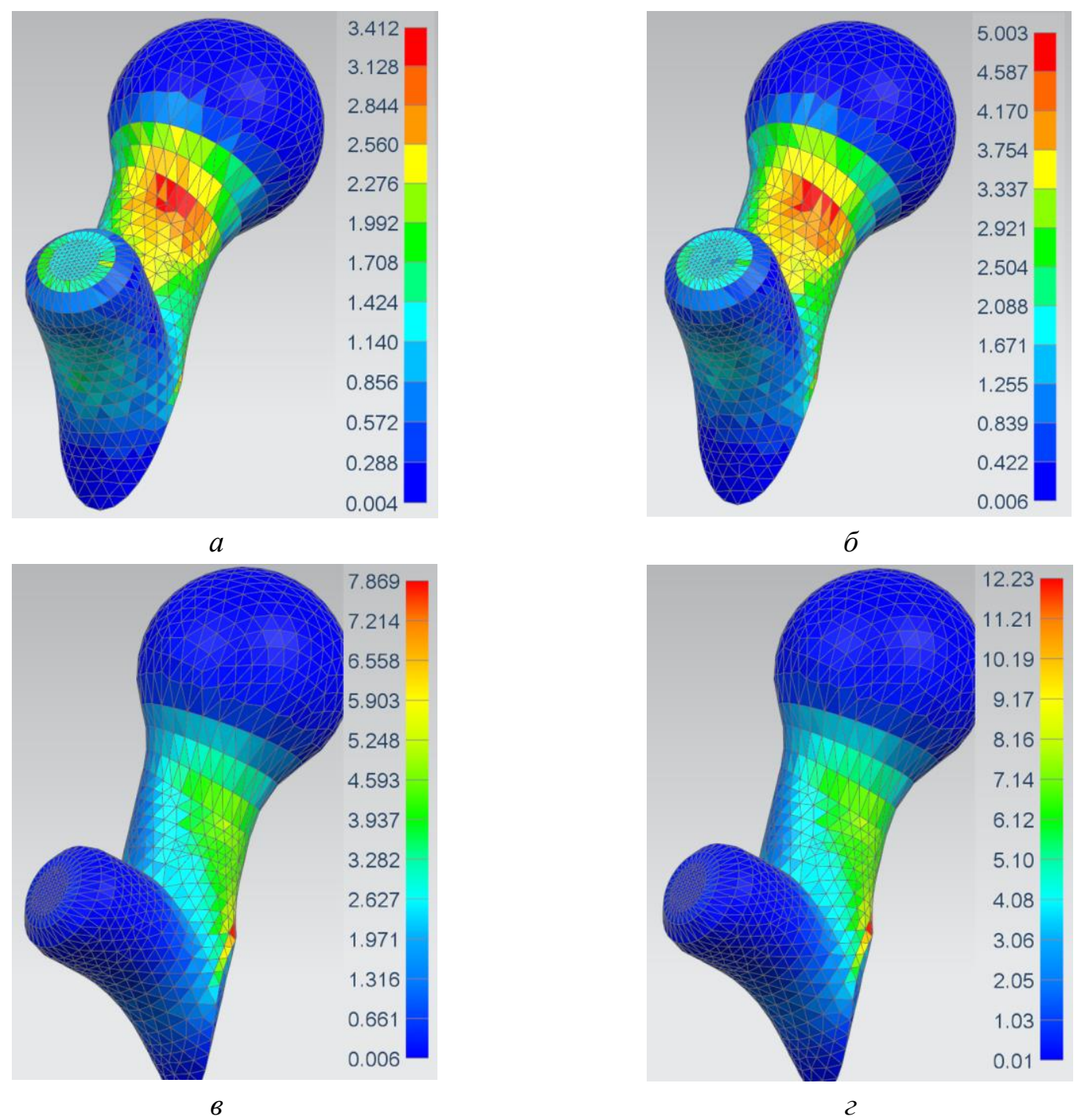

Рис. 8. Распределение максимальных касательных напряжений, МПа, в проксимальном участке бедренной кости при различных величинах угла ротации; $a$ - на $30^{\circ}$ вперед; $\sigma$ - на $50^{\circ}$ вперед; 8 - на $30^{\circ}$ назад, 2 - на $50^{\circ}$ назад

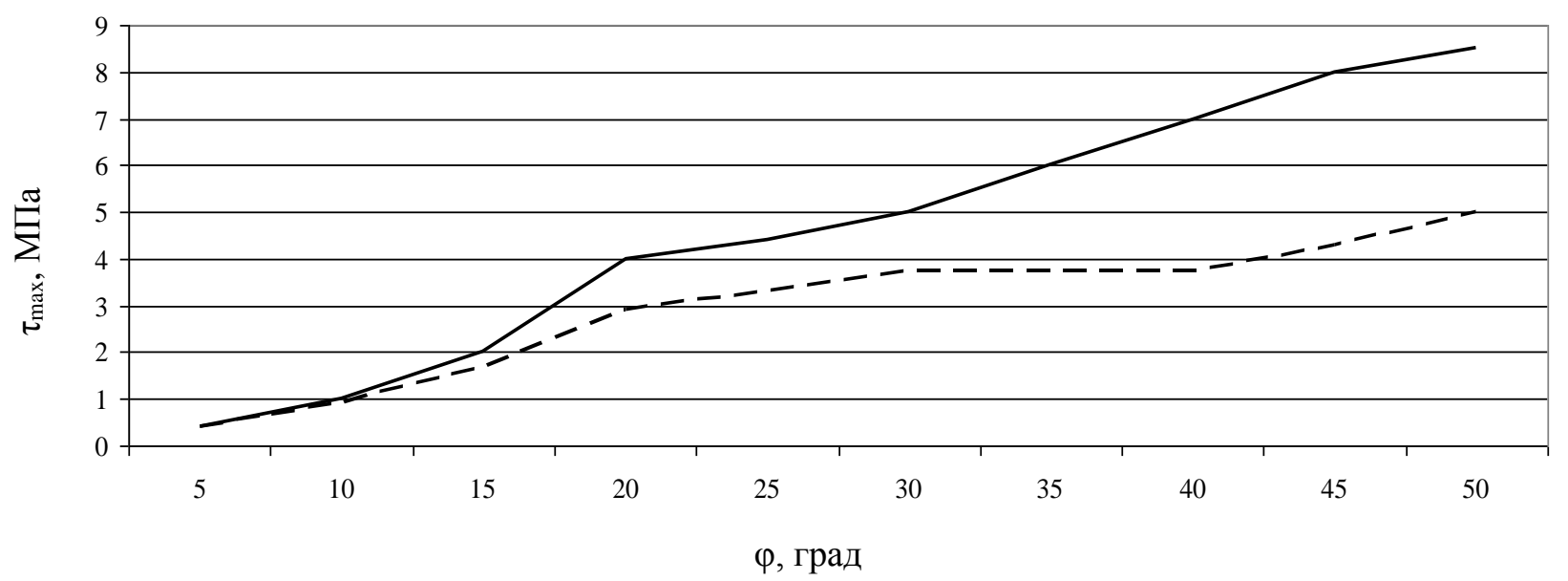

Рис. 9. Величины наиоольших максимальных касательных напряжений в шейке проксимального участка бедренной кости при различных величинах угла ротации: вращение вперед (пунктирная линия), вращение назад (сплошная линия) 
При анализе наибольших величин максимальных касательных напряжений в вертлужной впадине в зависимости от углов ротации (рис. 9) стоит отметить участок при ротации более $30^{\circ}$ назад. В этом случае величина напряжений превышает 6,4 МПа, что, согласно исследованиям [1-3], соответствует верхней границе предельных касательных напряжений. При ротации вперед наибольшие максимальные касательные напряжения достигают величины в 5,0 МПа.

Таким образом, при рассмотрении тактик ротации стоит отметить, что при вращении назад на величину угла более $30^{\circ}$ максимальные касательные напряжения попадают в нижнюю область критических значений, при $35^{\circ}-$ в верхнюю область критических значений. При рассмотрении ротации вперед на угол более $25^{\circ}$ максимальные касательные напряжения попадают в нижнюю область критических значений, перехода через верхнюю область критических значений при ротации до $50^{\circ}$ не происходит. Наибольшие величины максимальных касательных напряжений в вертлужной впадине при ротации в обоих направлениях до $50^{\circ}$ сильно не различаются, они же в проксимальном участке бедренной кости при ротации в различных направлениях на угол более $30^{\circ}$ начинают сильно различаться (более чем на $30 \%$ ).

\section{Выводы}

1. В работе приведены результаты численных исследований трехмерной модели таза при ротационной остеотомии, которые позволили оценить зависимость между усилиями, возникающими в мышцах, реактивной силой и реактивным моментом, действующими в суставе при различных углах ротации. Представлена картина распределения напряжений в вертлужной впадине и в проксимальном отделе бедренной кости при различной величине угла ротации. Полученные результаты позволяют судить о прочности костной ткани и качестве восстановления сустава.

2. При моделировании принимались следующие допущения: контактное взаимодействие считалось линейным; учитывались следующие мышцы: mm. piriformis, rectus femoris, iliopsoas, obturatorius internus, gluteus minimus, medius et maximus; они моделировались упругими элементами, работающими только на растяжение; рассечение для проведения ротационной остеотомии моделировалось параболическим разрезом; указанные допущения продиктованы необходимостью упрощения математической модели с сохранением ее адекватности. Фиксирующие элементы были исключены из расчетов.

3. На основании анализа результатов можно заключить, что безопасной величиной ротации при вращении назад являет угол до $30^{\circ}$, в этом случае наибольшие напряжения попадают в нижнюю границу предельных напряжений, при величине ротации более $35^{\circ}$ - в верхнюю границу предельных напряжений. При ротации вперед на величину более $25^{\circ}$ наибольшие напряжения попадают в нижнюю границу предельных напряжений, при ротации до $50^{\circ}$ верхняя граница предельных напряжений не достигается. Реальные величины допустимых напряжений могут быть уточнены для пациента в индивидуальном порядке.

4. Полученные результаты не являются абсолютно бесспорными, поскольку реальные условия во время хирургического вмешательства могут отличаться от заложенных в математическую модель, но проведенные исследования позволяют сформировать представление о влиянии величины угла ротации на компрессию в суставе. Это позволяет хирургу ориентироваться в вопросах выбора величины угла ротации и необходимости наложения дополнительных конструкций, которые помогают разгрузить тазобедренный сустав при значительных участках деструкции эпифиза головки бедренной кости. 


\section{БЛАГОДАРНОСТИ}

Работа выполнена при частичной финансовой поддержке Российского фонда фундаментальных исследований в рамках научных проектов № 16-04-00772, 15-3120602.

\section{СПИСОК ЛИТЕРАТУРЫ}

1. кулич А.Ю., Акулич Ю.В., Денисов А.С. Экспериментальное определение разрушающих касательных напряжений трабекулярной костной ткани головки бедра человека // Российский журнал биомеханики. - 2010. - Т. 14, № 4. - С. 7-16.

2. Акулич Ю.В., Акулич А.Ю., Денисов А.С. Влияние количества и размеров резьбовых фиксаторов на адаптационные изменения механических свойств губчатой костной ткани и усилие сжатия отломков после контролируемого остеосинтеза // Российский журнал биомеханики. - 2012. - Т. 16, № 2. С. 21-29.

3. Акулич Ю.В., Акулич А.Ю., Денисов А.С., Шайманов П.С., Шулятьев А.Ф. Уточнение индивидуальной зависимости модуля упругости трабекулярной костной ткани от объемного содержания матрикса // Российский журнал биомеханики. - 2014. - Т. 18, № 2. - С. 158-167.

4. Акулич Ю.В., Подгаец Р.М., Скрябин В.Л., Сотин А.В. Исследование напряженнодеформированного состояния эндопротезированного тазобедренного сустава // Российский журнал биомеханики. - 2007. - Т. 11, № 4. - С. 9-35.

5. Андреев П.С., Коноплев Ю.Г., Саченков О.А., Хасанов Р.Ф., Яшина И.В. Математическое моделирование ротационной флексионной остеотомии // Научно-технический вестник Поволжья. 2014. - № 5. - С. 18-21.

6. Балафендиева И.С., Бережной Д.В. Моделирование деформирования железобетонной обделки тоннеля в грунте с учетом одностороннего контактного взаимодействия ее блоков // Вестник Саратовского государственного технического университета. - 2011. - № 2 (55), вып. 1. - С. 8-16.

7. Банецкий М.В. Биомеханическое обоснование использования вертлужного компонента при эндопротезировании тазобедренного сустава: дис. ... канд.мед. наук. - М., 2008 - 94 с.

8. Бережной Д.В., Сагдатуллин М.К., Султанов Л.У. Расчет взаимодействия деформируемых конструкций с учетом трения в зоне контакта на основе метода конечных элементов // Вестник Казанского технологического университета. - 2014. - Т. 17, № 14. - С. 478-481.

9. Дещеревский В.И. Математические модели мышечного сокращения. - И.-М.: Наука, 1977. - 78 с.

10. Иванов Д.В., Барабаш А.П., Барабаш Ю.А. Интрамедуллярный стержень нового типа для остеосинтеза диафизарных переломов бедра // Российский журнал биомеханики. - 2015. - Т. 19, № 1. - С. 52-64.

11. Измайлова 3.T. Предоперационная диагностика модульной трансформации при чрескостном остеосинтезе бедренной кости // Российский журнал биомеханики. - 2009. - Т. 13, № 2. - С. 93-98.

12. Коноплев Ю.Г., Мазуренко А.В., Митряйкин В.И., Саченков О.А., Тихилов Р.М. Численное исследование влияния степени недопокрытия вертлужного компонента на несущую способность эндопротеза // Российский журнал биомеханики. - 2015. - Т. 19, № 4. - С. 330-343.

13. Климов О.В. Расчет и контроль биомеханической оси нижней конечности во фронтальной плоскости при ее коррекции по Илизарову // Российский журнал биомеханики. - 2014. - Т.18, № 2. С. 239-247.

14. Менщикова Т.И., Долганова Т.И., Аранович А.М. Влияние силы мышц бедра и голени на опорные реакции стоп у больных ахондроплазией после коррекции роста // Российский журнал биомеханики. 2014. - T. 18, N. 2. - C. 247-258.

15. Тверье В.М., Никитин В.Н. Задача коррекции прикуса в зубочелюстной системе человека // Российский журнал биомеханики. - 2015. - Т. 19, № 4. - С. 344-358.

16. Тверье В.М., Няшин Ю.И., Никитин В.Н. Биомеханическая модель определения усилий мышц и связок в зубочелюстной системе человека // Российский журнал биомеханики. - 2013. - Т. 17, № 2. C. $8-20$.

17. Шилько С.В., Черноус Д.А., Бондаренко К.К. Метод определения in vivo вязкоупругих характеристик скелетных мышц // Российский журнал биомеханики. - 2007. - Т. 11, № 1. - С. $45-54$.

18. Bennett J.T., Marurek R.T., Cash J.D. Chiarie's osteotomy in the treatment of Perthes Disease // J. Bone Jt. Surg. - 1991. - Vol. 73-B, № 2. - P. 225-228. 
19. Davydov R.L., Sultanov L.U. Numerical algorithm for investigating large elasto-plastic deformations // Journal of Engineering Physics and Thermophysics. - 2015. - Vol. 88 (5). - P. 1280-1288. DOI: 10.1007/s10891-015-1310-7.

20. Galiullin R.R., Sachenkov O.A., Khasanov R.F., Andreev P.S. Evalution of external fixation device stiffness for rotary osteotomy // International Journal of Applied Engineering Research. - 2015. - Vol. 10, № 24. - P. 44855-44860.

21. Heesakkers N., van Kempen R., Feith R., Hendriks J., Schreurs W. The long-term prognosis of LeggCalvé-Perthes disease: a historical prospective study with a median follow-up of forty one years // International Orthopaedics. - 2015. - Vol. 39 (5). - P. 859-863.

22. Nakamura N., Inaba Y., Machida J., Saito T. Rotational open-wedge osteotomy improves treatment outcomes for patients older than eight years with Legg-Calvé-Perthes disease in the modified lateral pillar B/C border or C group // International Orthopaedics. - 2015. - Vol. 39 (7). - P. 1359-1364.

23. Ozel B.D., Ozel D., Ozkan F., Halefoglu A.M. Diffusion-weighted magnetic resonance imaging of femoral head osteonecrosis in two groups of patients: Legg-Perthes-Calvé and avascular necrosis // Radiologia Medica. - 2016. - Vol. 121 (3). - P. 206-213.

24. Pailhe R., Cavaignac E., Murgier J., Cahuzac J.P., de Gauzy J.S., Accadbled F. Triple osteotomy of the pelvis for Legg-Calvé-Perthes disease: a mean fifteen year follow-up // International Orthopaedics. 2016. - Vol. 40 (1). - P. 115-122.

25. Sachenkov O., Kharislamova L., Shamsutdinova N., Kirillova E., Konoplev Yu. Evaluation of the bone tissue mechanical parameters after induced alimentary $\mathrm{Cu}$-deficiency followed by supplementary injection of $\mathrm{Cu}$ nanoparticles in rats // IOP Conference Series: Materials Science and Engineering. - 2015. Vol. 98. DOI:10.1088/1757-899X/98/1/012015.

26. Sachenkov O.A., Mitryaikin V.I., Zaitseva T.A., Konoplev Yu.G. Implementation of contact interaction in a finite-element formulation // Applied Mathematical Sciences. - 2014. - Vol. 8, № 159. - P. 7889-7897. DOI: 10.12988/ams.2014.49769.

27. Sagdatullin M.K., Berezhnoi D.V. Statement of the problem of numerical modelling of finite deformations // Applied Mathematical Sciences. - 2014. - Vol. 8, № 35. - P. 1731 - 1738. DOI: 12988/ams.2014.4283.

28. Sultanov L.U. Mathematical modeling of deformations of hyperelastic solids // Applied Mathematical Sciences. - 2014. - Vol. 8. - P. 7117-7124. DOI: 10.12988/ams.2014.49704.

29. Weiner S.D., Weiner D.S., Riley P.M. Pitfalls in treatment of Legg-Calvé-Perthes disease using proximal femoral varus osteotomy // J. Pediatr. Orthop. - 1991. - Vol. 11. - P. 20-24.

\section{NUMERICAL STUDY OF STRESS-STRAIN STATE OF PELVIS AT THE PROXIMAL FEMUR ROTATION OSTEOTOMY}

\section{O.A. Sachenkov, R.F. Hasanov, P.S. Andreev, Yu.G. Konoplev (Kazan, Russia)}

The relevance of this study is dictated by the high frequency of occurrence of the disease. Among all hip diseases, Legg-Calvé-Perthes disease takes $25-30 \%$ of diseases in childhood. There are no certain and founded recommendations for surgering in general and rotary flexion osteotomies in particular. The study is to determine the stress-strain state of the joint at different directions and magnitudes of the rotation angle. Calculations were carried out by using computer modelling of the hip joint based on localization of degenerative process and the severity of the degeneration of epiphysis in children and adolescents with Legg-Calvé-Perthes diseases. Numerical studies were performed using the finite element method in Nastran Siemens NX. The paper took into account the effect of the following muscles: mm. piriformis, rectus femoris, iliopsoas, obturatorius internus, gluteus minimus, medius et maximus. The calculations allow us to determine the forces produced in the muscles, reactive power and reactive torque acting in the joint at different rotation angles (these values are relevant for assessing the stiffness of external fixation device). 
The maximum shear stresses and Mises stresses have been identified in the acetabulum and the proximal part of the femur. Evaluation of strength was determined by the maximum shear stresses. So, in the acetabulum with a rotation of $25^{\circ}$ forward and rotation of $35^{\circ}$ back, the greatest value of maximum shear stress exceeds $1.7 \mathrm{MPa}$; at the rotation of more than $30^{\circ}$ back, the value of similar stress exceeds $6.4 \mathrm{MPa}$; at forward rotation to $50^{\circ}$, the highest maximum shear stresses reach a value of 5.0 MPa. Thus, when the rotation back to the angle over $30^{\circ}$, maximum shear stresses in the joint region are into the lower critical value, at 35 , $^{\circ}$ they are situated in the upper region of the critical values. When considering forward rotation angle over $25^{\circ}$, maximum shear stresses are into the lower region of the critical values, crossing through the upper region of the critical values at rotation to $50^{\circ}$ does not occur.

Key words: Legg-Calvé-Perthes disease, rotary flexion osteotomy, mathematical modelling.

Получено 7 апреля 2016 